\title{
Research on the Fault Feature Extraction Method of Rotor Systems Based on GAW-PSO
}

\author{
Feng Miao $\left(D^{1},{ }^{1}\right.$ Rongzhen Zhao, ${ }^{2}$ and Xianli Wang ${ }^{1}$ \\ ${ }^{1}$ School of Physical and Electrical Information, Luoyang Normal University, Luoyang 471022, China \\ ${ }^{2}$ Key Laboratory of Digital Manufacturing Technology and Application, The Ministry of Education, \\ Lanzhou University of Technology, Lanzhou 730050, China \\ Correspondence should be addressed to Feng Miao; miaofeng3699@163.com
}

Received 2 January 2020; Revised 2 February 2020; Accepted 10 February 2020; Published 9 March 2020

Academic Editor: Haopeng Zhang

Copyright (C) 2020 Feng Miao et al. This is an open access article distributed under the Creative Commons Attribution License, which permits unrestricted use, distribution, and reproduction in any medium, provided the original work is properly cited.

In order to solve the problem of blind separation of signals from dynamic hybrid rotor systems, this paper proposed an improved adaptive inertial weight particle swarm optimization method based on genetic mechanism. The method takes the negative entropy of separated signal as the objective function and adaptively adjusts the inertia weight according to the difference of particle fitness, thus reducing the number of invalid iterations. At the same time, genetic hybridization mechanism was introduced to increase population diversity and facilitate the processing of dynamic mixed signals. The orthogonal matrix is expressed as a parameterized form, which can reduce the complexity of the algorithm. The simulation results showed that the performance of the proposed method is better than that of the traditional method for blind separation of dynamic hybrid analog mechanical signals. It can separate the actual dynamic rotor system signals and achieve the purpose of fault feature extraction.

\section{Introduction}

During the operation of rotating machinery, the vibration signals measured by using vibration sensors are often superimposed by the vibrations of multiple components. How to analyse, process, and identify these signals is extremely important to judge and diagnose the working state of rotating machinery. The direct analysis and processing of sensor signals is bound to cause great difficulties in mechanical condition monitoring and fault diagnosis [1-3].

Various traditional modern signal processing methods, such as Fourier transform, short-time Fourier transform, and wavelet transform, have been widely used in vibration signal analysis, but the above analysis methods have obvious disadvantages to many kinds of mixed vibration signals on rotating machinery $[2,4]$. Blind source separation technology can realize the separation of multialiasing signal, while it is not affected by the overlap of source signal time and spectrum and the output signal after separation will not lose the weak feature information in the source signal [4]. In [5], the blind separation algorithm, empirical modal decomposition, and wavelet packet transform are combined to process the mechanical multifault signal. $\mathrm{Li}$ et al. [6] obtained the judgment criteria through the subjective Bayesian derivation and these were applied to multisensor distributed detection fusion algorithm to solve the problem of uncertainty in mechanical signal detection. The literature [7] was proposed to apply the mean pattern decomposition and blind separation algorithm of the extreme domain to single-channel mechanical fault detection at the same time. The literature [8] used particle swarm algorithm combined with blind separation algorithm for mechanical fault diagnosis. However, the traditional blind separation method of rotor signal is processed under the condition where the source signal is stable and the mixed channel is static, and the observation signal is mixed in the stochastic timevarying channel when the actual fault signal is separated blindly. Therefore, it is more practical to solve the problem of blind separation of random dynamic mixed fault signals in nonstationary environment.

In order to solve the problem of blind separation of stochastic dynamic mixed fault signal in the nonstationary 
environment, a method for blind separation of dynamic fault signal is proposed. On the basis of particle swarm optimization (PSO), the inertia weight is adjusted adaptively and optimized by genetic algorithm (GA). The inertia weight of the algorithm will adjust adaptively with the difference between the fitness before and after each particle iteration, eliminating the adverse effect of the particle inertia component on the next iteration, which can reduce the number of invalid iterations and accelerate the convergence speed. At the same time, the genetic hybridization mechanism is introduced to increase the population diversity, so that the particles are not easy to fall into the local optimum, which can effectively solve the instability problem of blind separation of random dynamic mixed rotor vibration signals. On this basis, the separation orthogonal matrix is represented in the form of parameterization, which can effectively reduce the complexity of the algorithm. The experimental results show that the algorithm can effectively separate the random dynamic mixed rotor vibration signals, estimate the source signals well in time and frequency domain, and have better separation effect than the traditional algorithm; the algorithm can also effectively separate the actual dynamic rotor fault signals and then can achieve the detection of rotor coupling fault.

\section{Blind Source Separation Model}

Blind source separation (BSS) refers to estimating the parameters of each transmitter and transmission channel only by observing the signals when the parameters of each input signal and transmission channel are unknown $[9,10]$. The mixing process is expressed by mathematical formulas as follows:

$$
x(t)=A s(t)+n(t) .
$$

In formula $(1), x(t)=\left[x_{1}(t), x_{2}(t), \ldots, x_{M}(t)\right]^{T}$ is a $\mathrm{M}$-dimensional random observation vector with noise, $A$ is a mixed matrix of unknown full rank $M \times N, s(t)=$ $\left[s_{1}(t), s_{2}(t), \ldots, s_{N}(t)\right]^{T}$ is a $N$-dimensional source signal, and $s_{i}(t)$ of each component in the source signal is assumed to be statistically independent, and it contains at most one Gaussian noise; otherwise, it cannot be separated; $n(t)=$ $\left[n_{1}(t), n_{2}(t), \ldots, n_{M}(t)\right]^{T}$ is a M-dimensional noise signal:

$$
y(t)=W x(t)
$$

The purpose of blind source separation is to find a separation matrix $W$, so that $y(t)=W x(t)$ is the optimal estimate of $s(t)$.

Since blind source separation estimates the input signal only according to the observed signal, without any prior knowledge about the source signal, there are some uncertainties between the estimated input signal and the source signal, which are mainly reflected in the uncertainties in the estimation of the amplitude and order of the input signal. But these two uncertainties do not affect the analysis of the signal, because most of the information of the signal is contained in the waveform rather than in the magnitude and order $[11,12]$.

\section{Adaptive Particle Swarm Algorithm for Improved Genetic Hybridization Mechanism}

3.1. Particle Swarm Optimization. Particle swarm optimization (PSO) initializes the particle population and calculates the fitness value from the objective function to make the particle move to the optimal position $[13,14]$. According to the motion state and fitness function of particles, the velocity and position of particles are updated iteratively, and the global optimal position is finally found. The velocity and position update formulas are as follows:

$$
\begin{aligned}
V_{i, j}(t+1) & =V_{i, j}(t)+c_{1} r_{1}\left[p_{i, j}-X_{i, j}(t)\right]+c_{2} r_{2}\left[p_{g, j}-X_{i, j}(t)\right], \\
X_{i, j}(t+1) & =X_{i, j}(t)+V(t+1), \\
j & =1,2, \ldots, D .
\end{aligned}
$$

In formula (3), $c_{1}$ and $c_{2}$ are the learning factors, $r_{1}$ and $r_{2}$ are the random numbers distributed in $[0,1], D$ is the dimension of particle search space, $X_{i}=\left(X_{i, 1}, X_{i, 2}, \ldots, X_{i, D}\right)$ is the $i$ th particle position, $V_{i}=$ $\left(V_{i, 1}, V_{i, 2}, \ldots, V_{i, D}\right)$ is the $i$ th particle velocity, $P_{i}=\left(p_{i, 1}, p_{i, 2}, \ldots, p_{i, D}\right)$ is the optimal position of individual particle, and $P_{g}=\left(p_{g, 1}, p_{g, 2}, \ldots, p_{g, D}\right)$ is the global optimal position of current population.

Shi first applied the inertia weight $\omega$ to the PSO algorithm. The inertia weight directly affects the update speed of particles and the convergence performance of the algorithm $[12,15]$ :

$$
\begin{aligned}
V_{i, j}(t+1)= & \omega(t) V_{i, j}(t)+c_{1} r_{1}\left[p_{i, j}-X_{i, j}(t)\right] \\
& +c_{2} r_{2}\left[p_{g, j}-X_{i, j}(t)\right] .
\end{aligned}
$$

In formula (4), $\omega$ is the inertial weight. The inertial weight value can be adjusted to achieve an effective balance between particle search ability and convergence speed. Inertial weights can be reduced in the linear mode, that is, within $[0.96,0.5]$. This makes the early inertial weight larger, have the good global search ability, and not easy to fall into the local optimal. Although the late inertial weight is small, it has the good local search ability and can accelerate the convergence:

$$
\omega(t)=\omega_{\max }-\left(\omega_{\max }-\omega_{\min }\right) \times(t N) .
$$

In formula (5), $\omega(t)$ is the inertia weight at the $t$ th iteration, $N$ is the maximum iteration number, $\omega_{\max }$ is the maximum inertia weight, and $\omega_{\min }$ is the minimum inertia weight.

\subsection{Genetic Algorithm Improved Adaptive Inertia Weight} Particle Swarm Optimization. After each iteration, some of the particles will reach a better fitness position, while others will reach a suboptimal or worse position. In the next iteration, the particle with the best position will reach the better position after iteration, while the particle with the suboptimal or the worse position will reach the worse position after iteration. The particle with the best final position will approach or reach the global optimum position after 
many iterations, while the other particles will do the opposite. Therefore, when the particle is updated each time, the inertia weight of each particle can be dynamically adjusted according to the fitness value of the last iteration, which is very helpful to the global optimization and fast convergence of the particle population.

According to the above discussion, PSO algorithm can be improved by adaptive inertia weight. Based on the difference of fitness before and after iteration, the whole particle swarm is divided into two parts, that is, the part with better fitness after iteration and the part with worse fitness. For those particles whose position is better, the inertia weight is set to the linear decreasing mode (formula (5)) to ensure that the population can converge quickly and optimize globally. For the other part of particles whose position is worse, the inertia weight is set to 0 , which eliminates the adverse effects of inertia component on particle velocity and position updating $[16,17]$ :

$$
\delta f\left[X_{i}(t)\right]=f\left[X_{i}(t)\right]-f\left[X_{i}(t-1)\right] .
$$

In formula (6), $i=1,2, \ldots, n, n$ is the number of particles; $t \geq 2 ; f\left[X_{i}(t)\right]$ is the fitness value of the $i$ particle in the $t$ iteration; and $\delta f\left[X_{i}(t)\right]$ is the difference between the fitness of the $i$ particle in the $t$ iteration and the $t-1$ iteration. In the current iteration, the dynamic adjustment values of inertia weights of each particle are expressed as follows:

$$
\omega_{i}(t)= \begin{cases}w(t), & \delta f\left[X_{i}(t)\right] \geq 0 \\ 0, & \delta f\left[X_{i}(t)\right] \leq 0\end{cases}
$$

The adaptive inertia weight particle swarm optimization (AW-PSO) is obtained by substituting formula (7) into formula (4). The AW-PSO algorithm has good effect on blind separation of static signals and can quickly and accurately search for the global optimum. However, when applied to blind separation of dynamic fault mechanical signals, although the convergence speed is fast, it is difficult to find the global optimum of the population $[18,19]$. Therefore, referring to the concept of genetic hybridization, this paper sets the proportion of hybridization $S_{p}$ and chooses the corresponding particle population total $M$ random pairwise hybridization, in which $S_{p} * M$ is the number of particles selected for hybridization. The updating formulas of position and velocity hybridization are as follows:

$$
\left\{\begin{array}{l}
X_{m}^{c}(t)=p_{c} X_{m}(t)+\left(1-p_{c}\right) X_{n}(t), \\
X_{n}^{c}(t)=\left(1-p_{c}\right) X_{m}(t)+p_{c} X_{n}(t) .
\end{array}\right.
$$

In formula (8), $p_{c}$ is the probability of genetic hybridization; in the $t$ th iteration, the offspring particles $X_{m}^{c}$ and $X_{n}^{c}$ are obtained by the arithmetic hybridization of the parent particles $X_{m}$ and $X_{n}$. The parent particle velocity can be obtained by the following formula:

$$
\left\{\begin{array}{l}
X_{m}^{c}(t)=\frac{V_{m}(t)+V_{n}(t)}{\left|V_{m}(t)+V_{n}(t)\right|}\left|V_{m}(t)\right|, \\
X_{n}^{c}(t)=\frac{V_{m}(t)+V_{n}(t)}{\left|V_{m}(t)+V_{n}(t)\right|}\left|V_{n}(t)\right| .
\end{array}\right.
$$

After each iteration, the part of the particle population with better fitness is processed by formulas (8) and (9) to obtain new particle positions and velocities, which can increase the diversity of the population and better facilitate blind separation of dynamic signals. This is the genetic algorithm improved adaptive inertia weight particle swarm optimization (GAW-PSO).

\section{Blind Separation Algorithm Based on GAW-PSO}

4.1. Objective Function. According to information theory, the entropy of Gauss variable is the largest among all equal variance random variables, so entropy can be used to measure non-Gauss. According to the central limit theorem, if a random variable $X$ consists of the sum of many independent random variables $S_{i}$, as long as $S_{i}$ has a finite mean and variance, then regardless of its distribution, the random variable $X$ is closer to the Gaussian distribution than $S_{i}$. In other words, $S_{i}$ is more non-Gaussian than $X$. Therefore, in the separation process, we can express the mutual independence of the separation results by the non-Gaussian measurement of the separation results. When the nonGaussian measurement reaches the maximum, it indicates that the separation of the independent components has been completed [13].

According to the central extremum theorem, the fitness function of the GAW-PSO is defined by using the negative entropy of separated signals. Negative entropy is used to measure the independence of each separated signal. When the negative entropy is maximum, the non-Gaussian property between separated signals is also the strongest [20]. When the probability distribution of the separated signal is symmetrical, the negative entropy of the first separated signal can be approximately expressed as

$$
J_{i}\left(y_{i}\right) \cong \frac{1}{48} k_{4}^{2}\left(y_{i}\right)
$$

where $k_{4}\left(y_{i}\right)$ is the fourth-order cumulant of the $i$-way separated signal, namely kurtosis, which can be normalized and expressed as

$$
k_{4}\left(y_{i}\right)=\frac{E\left(y_{i}^{4}\right)}{E\left(y_{i}^{2}\right)^{2}}-3 .
$$

In order to satisfy the constraints of zero mean and $E\left(y y^{T}\right)=I$, centralization and prewhitening of the separated signal are needed before calculating the negative 
entropy. Therefore, the fitness function of the GAW-PSO algorithm is expressed as

$$
f(y)=\sum_{i=1}^{n} \frac{1}{48} k_{4}^{2}\left(y_{i}\right)
$$

Under the constraint of $E\left(y y^{T}\right)=I$, if the fitness function becomes larger, the separation effect is better.

4.2. Parametric Representation of Orthogonal Matrix. According to the constraints on the separated signal in equation (12), the successful separation of $y(t)$ can be guaranteed, as long as the separation matrix satisfies the orthogonal condition in the separation process. When dealing with $n$-order matrices, $n^{2}$ unknowns need to be solved and the computational complexity is large. Because the degree of freedom of $n$-order orthogonal matrices is $n(n-1) / 2$, the parameterized representation of $N$-order orthogonal matrices can significantly reduce the computational complexity. Through Cayley transformation, any $\mathrm{N}$ order orthogonal matrix whose determinant is not equal to 1 can be transformed into the product form of rotation matrix. The orthogonal matrix is $B_{2 \times 2}(\theta)=[\cos \theta .-\sin \theta ; \sin \theta, \cos \theta]$, expressed by parameter vector $\theta=\left\{\theta_{1}, \theta_{2}, \ldots, \theta_{n(n-1) / 2}\right\}$, in which parameter vector $\theta \in[0,2 \pi]$.

4.3. Blind Separation Method Based on GAW-PSO. GAW-PSO algorithm uses particle position to represent the orthogonal separation matrix, which can reduce the dimension $D$ of particle search from $n^{2}$ to $n(n-1) / 2$ by parameterized representation, thus improving the efficiency of the algorithm. Furthermore, GAW-PSO algorithm adaptively chooses inertia weight to make the particle move to the global optimal position and introduces genetic hybridization mechanism to improve the diversity of the population, which can effectively blind separation dynamic signals. The specific steps are as follows:

(1) First, the observation dynamic mixed fault mechanical signal $x(t)$ is sampled, and the centralization and prewhitening treatment are carried out.

(2) Particle population initialization randomly generates the separation matrix as the initial position of each particle and randomly initializes the velocity of each particle at the same time. The learning factors $c_{1}$ and $c_{2}$, maximum inertia weight $\omega_{\max }$, and minimum inertia weight $\omega_{\min }$ are initialized, respectively.

(3) $y(t)$ can be calculated according to the sum of separation matrix (2), and then, the fitness values of each particle can be calculated by the sum of formulas (11) and (12).

(4) Update $P_{i}$ and $P_{g}$ iteratively according to fitness value.

(5) When $t \geq 2$, the inertia weight of each particle is adaptively adjusted by formulas (6) and (7).

(6) According to the probability of genetic hybridization, the corresponding number of particles is selected to produce the same number of offspring particles by random hybridization. The position and velocity of the offspring are determined by formulas (8) and (9).

(7) The inertia weights obtained in step 5 are substituted into formula (4), and the velocity and position of each particle are updated iteratively in step 6 .

(8) Enter the loop state and output the result when the loop termination condition is reached; otherwise, return to step 3 to continue the search.

\section{Simulation and Experimental Analysis}

5.1. Evaluation Criteria. In order to effectively evaluate the effect of signal separation, the similarity coefficient $\xi_{i j}$ is used as the evaluation index of the difference between the separated signal and the source signal [21, 22]. The similarity coefficient is defined as

$$
\xi_{i j}=\xi\left(y_{i}, s_{j}\right)=\frac{\left|\sum_{t=1}^{M} y_{i}(t) s_{j}(t)\right|}{\sqrt{\sum_{t=1}^{M} y_{i}^{2}(t) \sum_{t=1}^{M} s_{j}^{2}(t)}} .
$$

If the similarity coefficient matrix has only one element close to 1 in each row and column and the other elements are close to 0 , then the separation effect of the algorithm is ideal.

5.2. Simulations. In the event of friction, crack, and no medium fault in the rotor system, the signals collected by the sensor often appear as mixed fault signal and amplitude modulation signal. In the simulation test, four kinds of signals are selected, and the production function of the source signal is as follows:

$$
s(t)=\left(\begin{array}{c}
s_{1}(t) \\
s_{2}(t) \\
s_{3}(t) \\
s_{4}(t)
\end{array}\right)=\left(\begin{array}{c}
n(t) \\
3 \sin 0.4 t \cos 10 t \\
\sin 3 t+\sin 6 t+\sin 10 t \\
\sin 2 t
\end{array}\right) .
$$

In equation (14), source signal $s_{1}(t)$ simulates periodic vibration signal; $s_{2}(t)$ simulates misalignment fault characteristic signal; $s_{3}(t)$ simulates rubbing fault characteristic signal; and $s_{4}(t)$ simulates noise signal. The time-domain waveform of $s_{1}(t), s_{2}(t), s_{3}(t), s_{4}(t)$ is shown in Figure 1 . The source signal generates the observed signal $x_{1}(t), x_{2}(t), x_{3}(t)$, and $x_{4}(t)$ by random mixing. The mixing mode is linear superposition. The time-domain waveform of the mixed signal is shown in Figure 2.

In the simulation experiment, the particle swarm algorithm and the GAW-PSO algorithm are used to separate the mixed signals, and the separation signals are shown in Figures 3 and 4, respectively.

Compared with Figures 3 and 4, it can be seen the source signals separated by the general particle swarm optimization algorithm cannot reflect the waveform information of the source signals; the source signals separated by the improved adaptive particle swarm optimization algorithm can well reflect the waveform information of the source signals. 

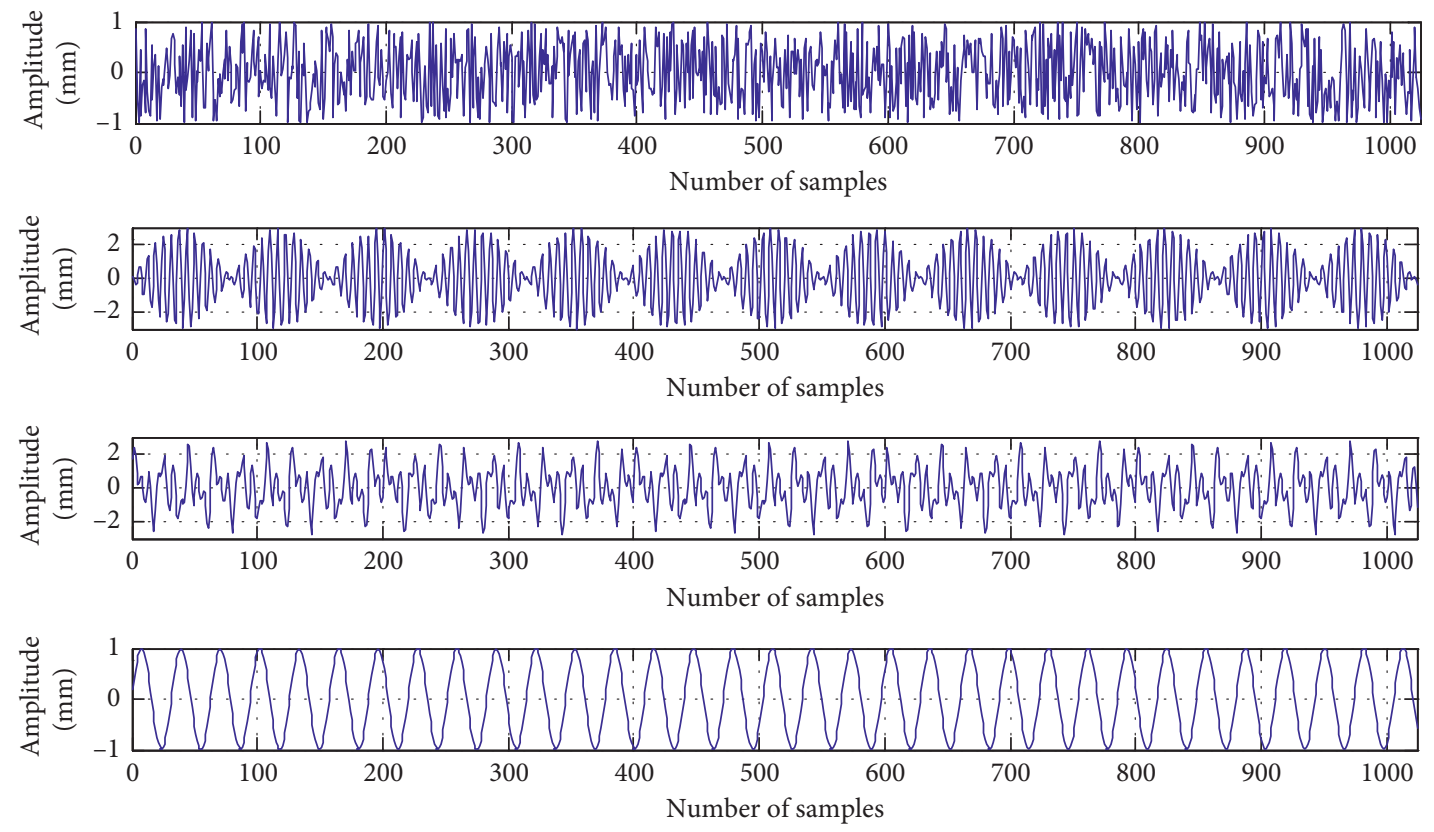

Figure 1: The simulative signal.
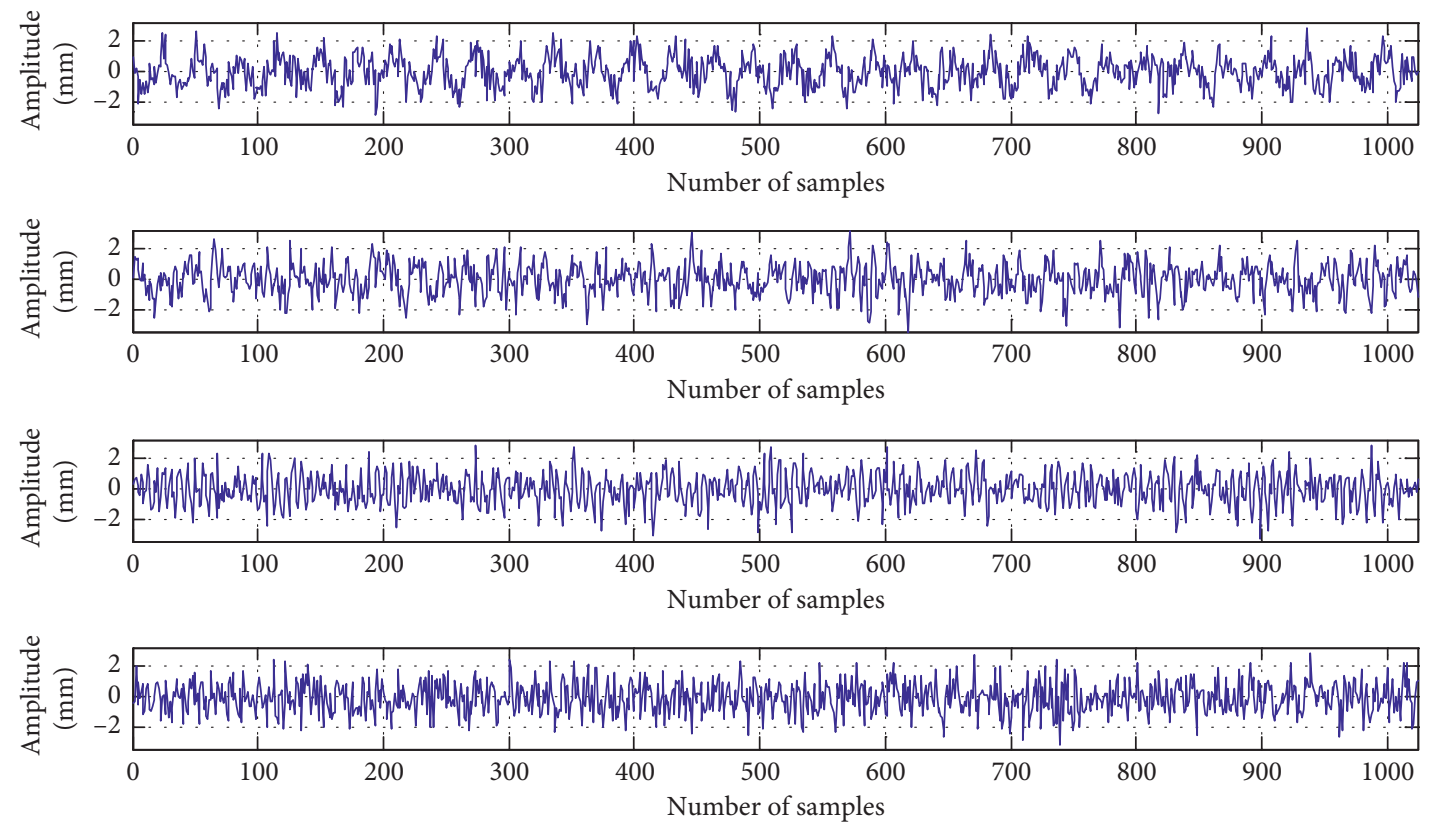

FIgURE 2: The mixture of simulative signal.

Although the amplitude and sequence of the source signals and the separated signals are inconsistent, it does not affect the recognition of signal characteristics. The simulation experiment quantitatively evaluates the similarity of the improved adaptive particle swarm optimization algorithm to separate the signal from the source signal by using a correlation coefficient. The similarity coefficient matrix is as shown in Table 1.

The underline coefficient is expressed as the correlation coefficient between the separated signal and the source signal. From the similarity coefficient, it can be seen that the similarity coefficient between the separated signal and the source signal based on the improved particle swarm optimization algorithm is very high, which shows that the improved adaptive particle swarm optimization algorithm can accurately extract the characteristics of the source signal from the mixed signal.

5.3. Experiments. In order to verify the separation performance of the above algorithm for the measured vibration signals of the aliasing rotor, this paper analyses the vibration signals of the aliasing rotor. The rotor system and test bench are shown in Figure 5. In the process of rotor rotation, there 

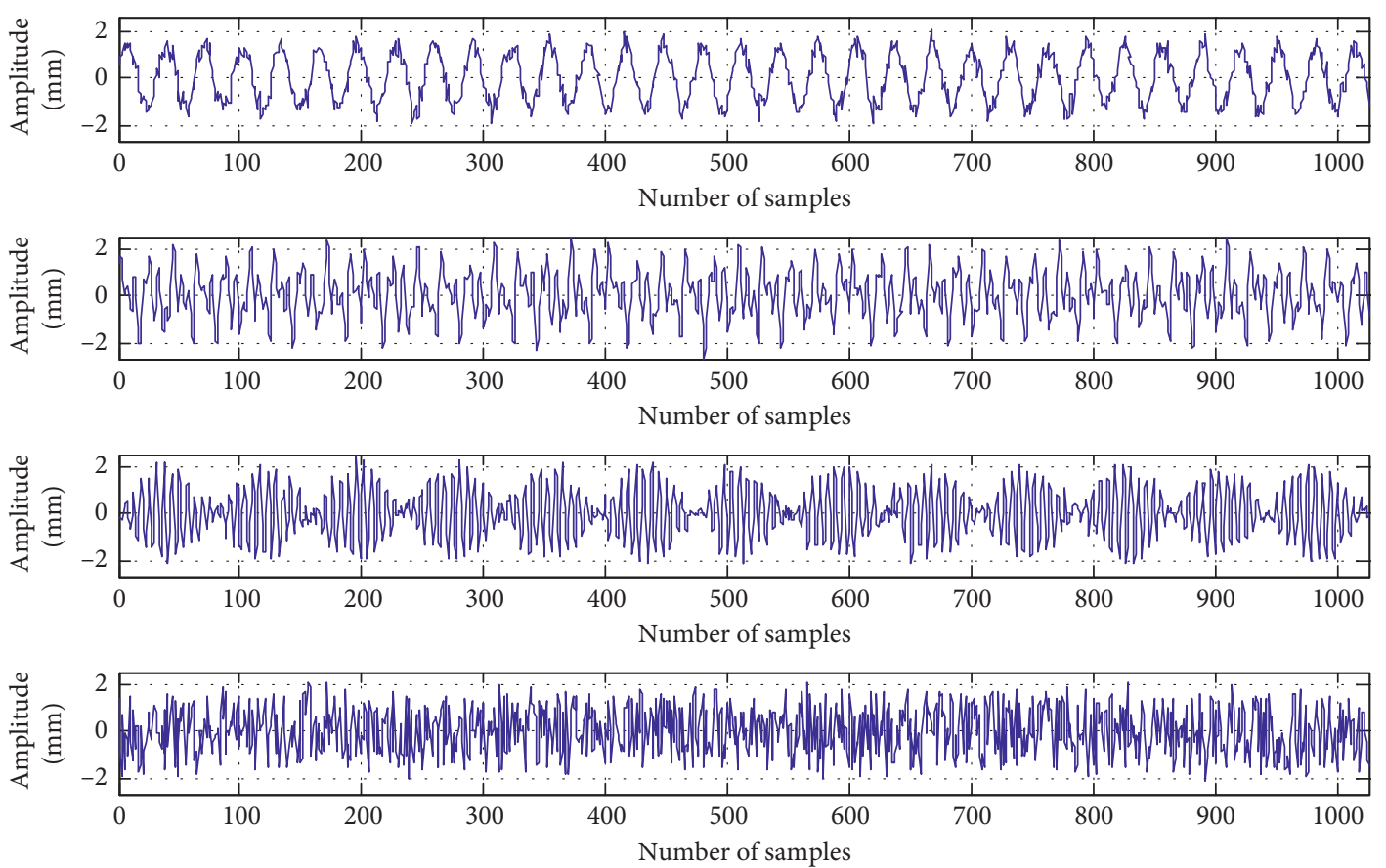

FIgURE 3: The separated signal using PSO.
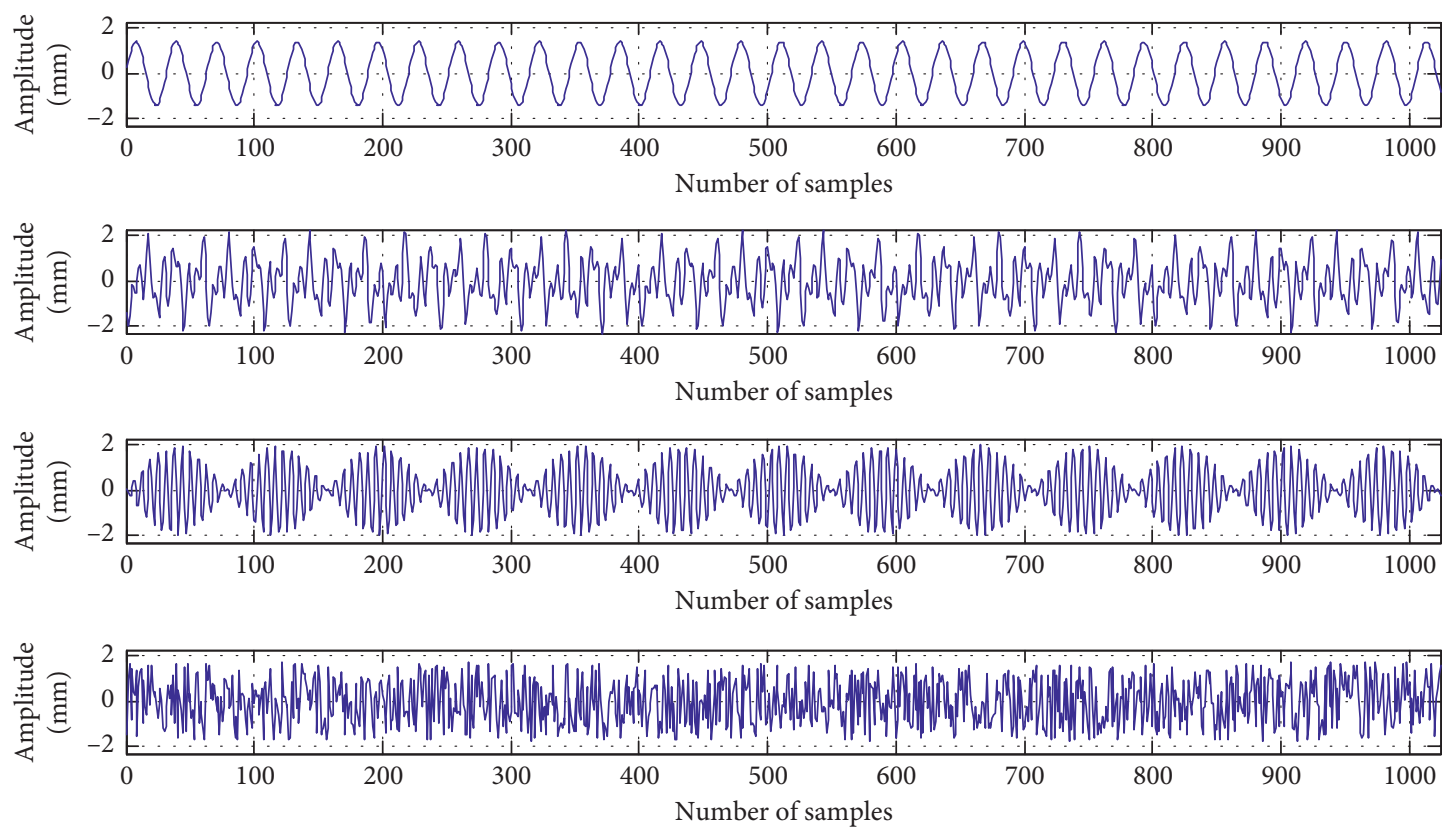

FIgure 4: The separated signal using GAW-PSO.

TABLe 1: Similarity coefficient and time of two algorithms.

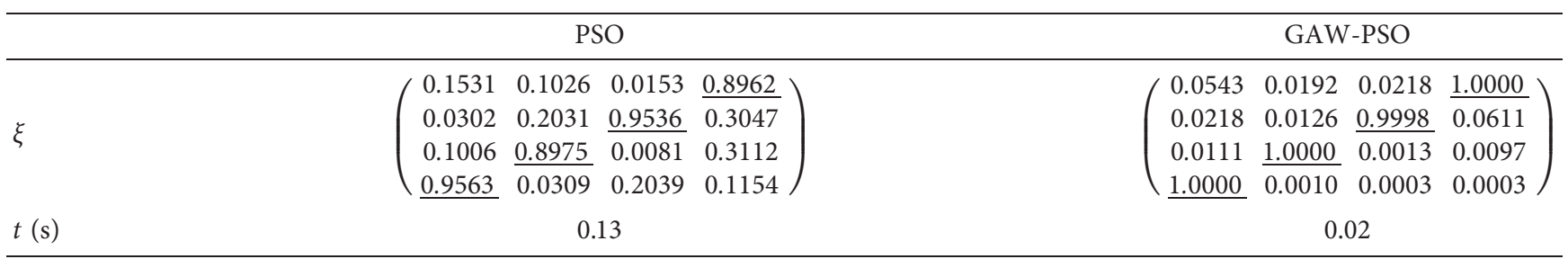




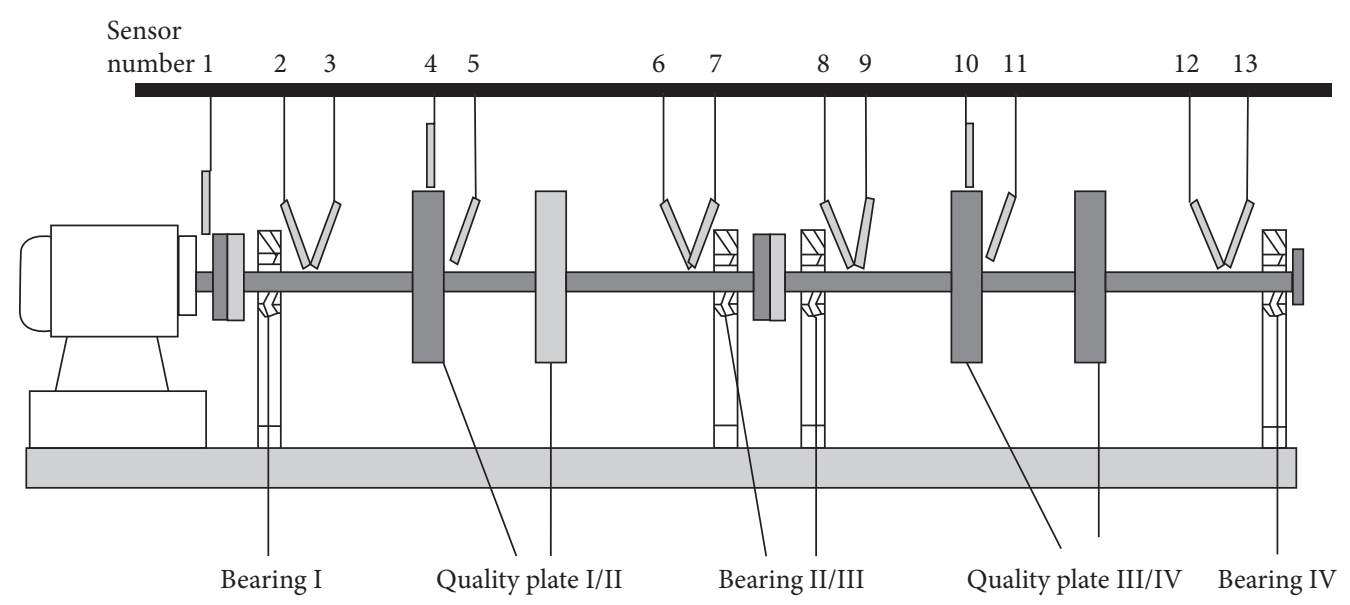

Figure 5: Position of the sensor on the test bench.
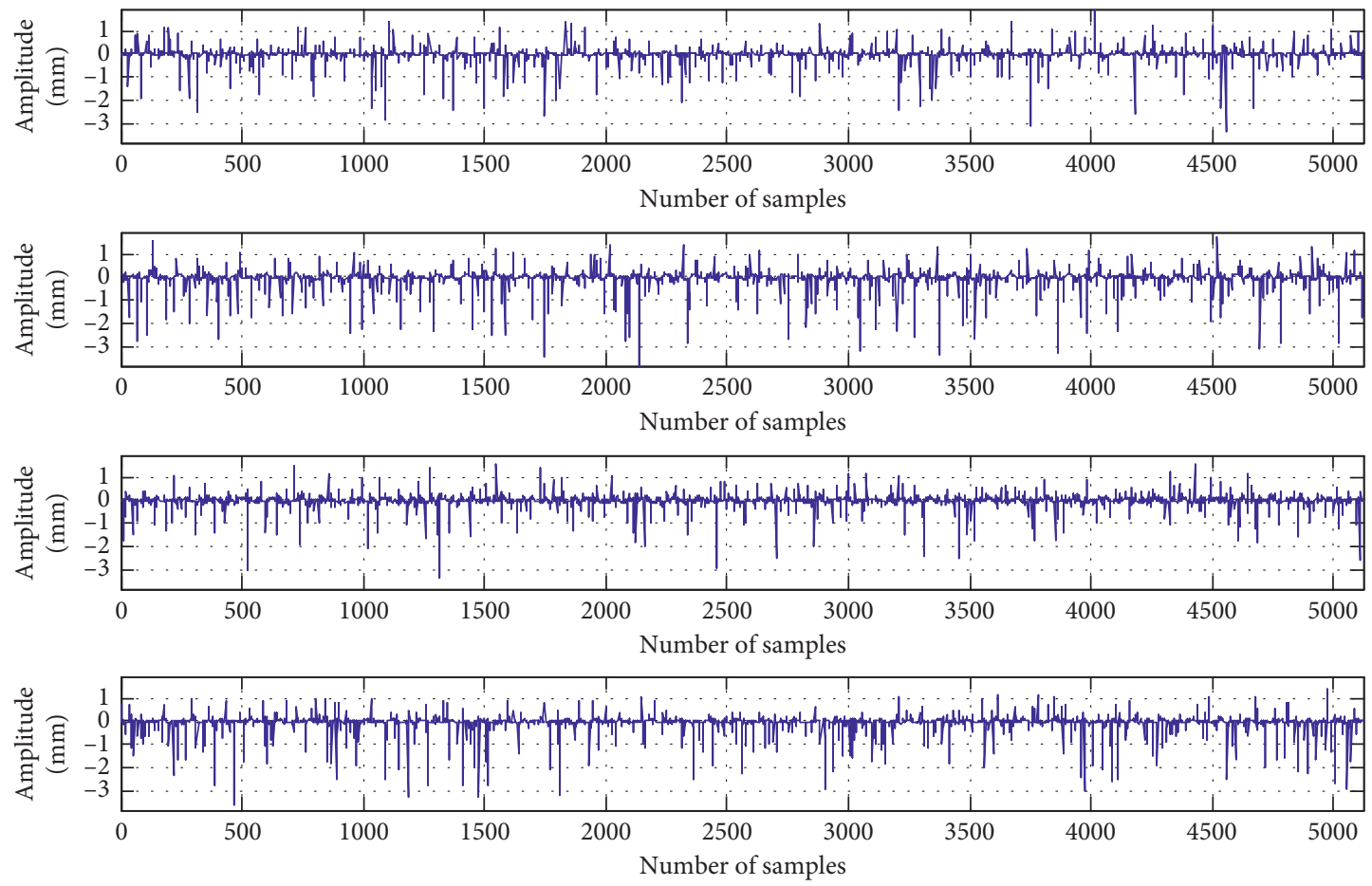

FIgURE 6: The vibration signal for rotor with multifaults.

may be many potential source signals, such as bearing ball vibration signal, shaft axial vibration signal, and noise signal, and the sensor carries out measurements at the same time, so the signal measured by the sensor is a mixed vibration signal. In order to satisfy the hypothesis that the number of sensors in blind source separation (BSS) is greater than or equal to the number of source signals, the signals labeled 2, 3, 4, and 5 are used in the experiment. The rotating speed of the rotor is about $2800 \mathrm{r} / \mathrm{min}$, and the sampling frequency is $5000 \mathrm{~Hz}$. The measured four sensor signals are shown in Figure 6. The time-domain signals separated directly by the improved algorithm are shown in Figure 7 . In order to compare the complex vibration of the rotor before and after separation more intuitively, the authors carried out the spectrum analysis of the data signals and observed the different characteristics of the signals from the frequency domain before and after separation. The spectra before and after separation are shown in Figures 8 and 9.

In Figure 8, because the characteristic frequency of the fault overlaps on each power spectrum, it is difficult to determine the existence of the fault type and make accurate fault diagnosis. Because the system is a rigid structure, the vibration delay can be ignored and the transient model can be maintained. Therefore, the GAW-POS method is used to separate the fault signal and obtain the separated signal. The power spectrum characteristics are shown in Figure 9.

As can be seen from Figure 9, in the separated signals, the power spectrum of each signal basically displays only one 

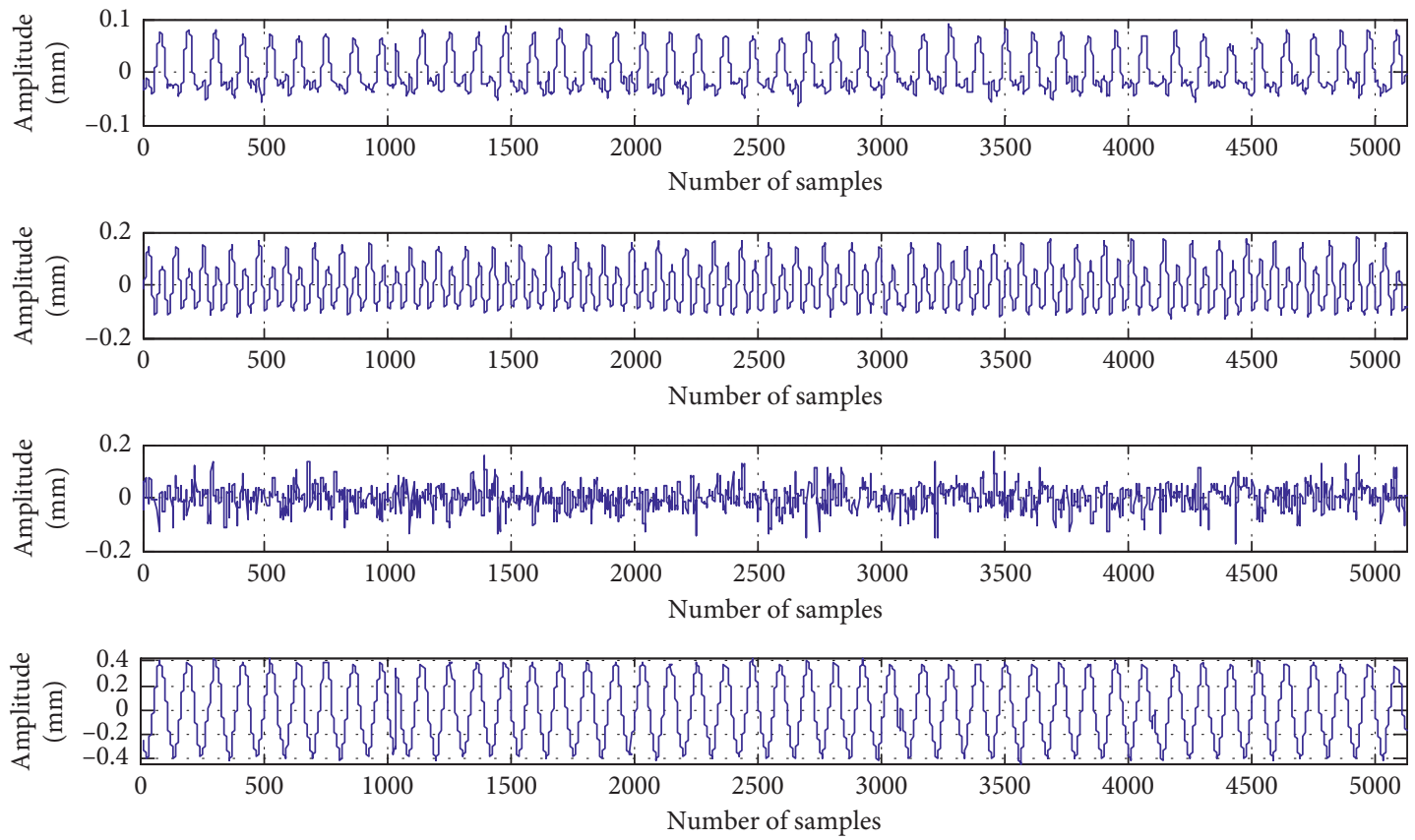

Figure 7: The separated signal using GAW-PSO.
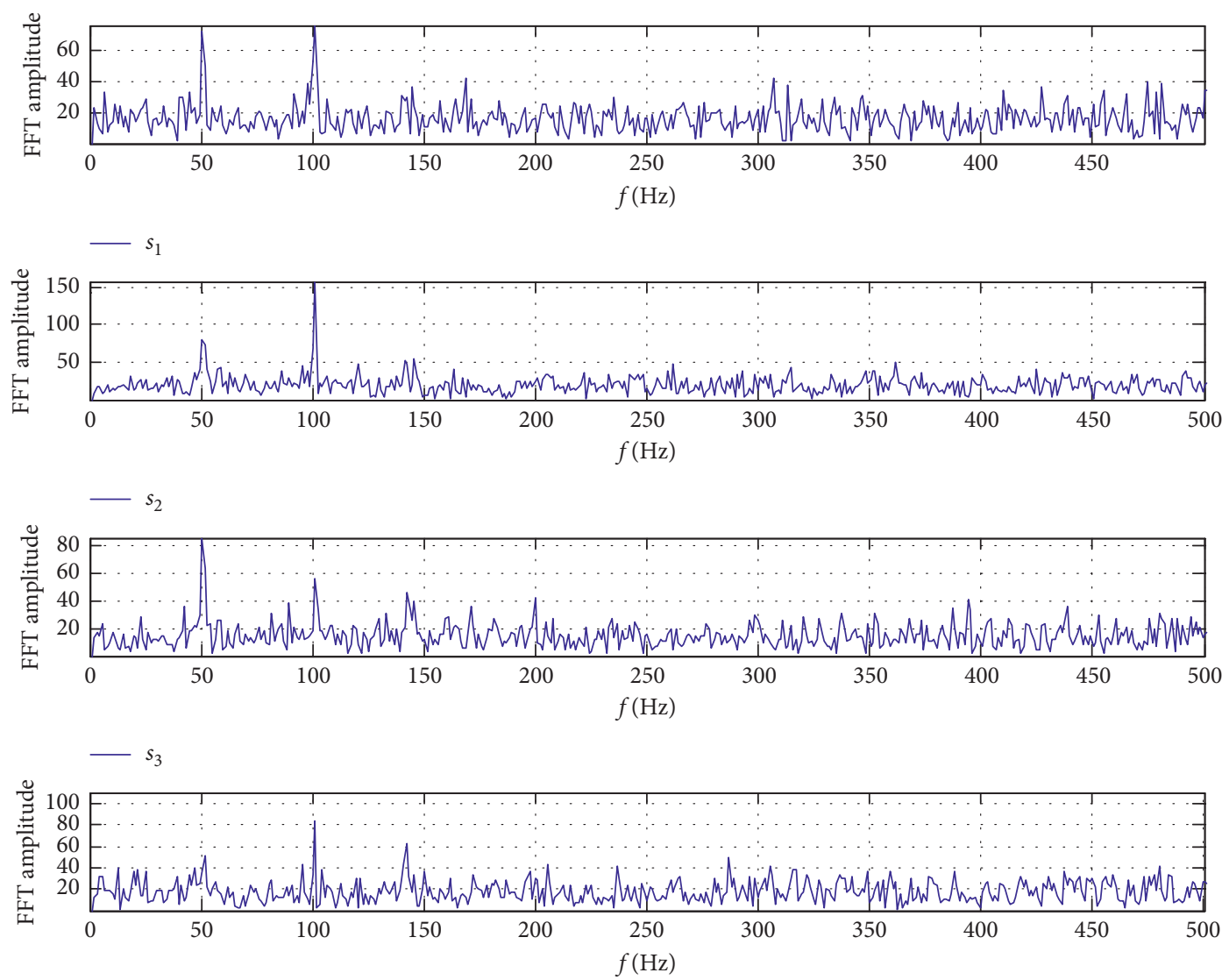

FIgURE 8: FFT of vibration signal for rotor with multifaults. 

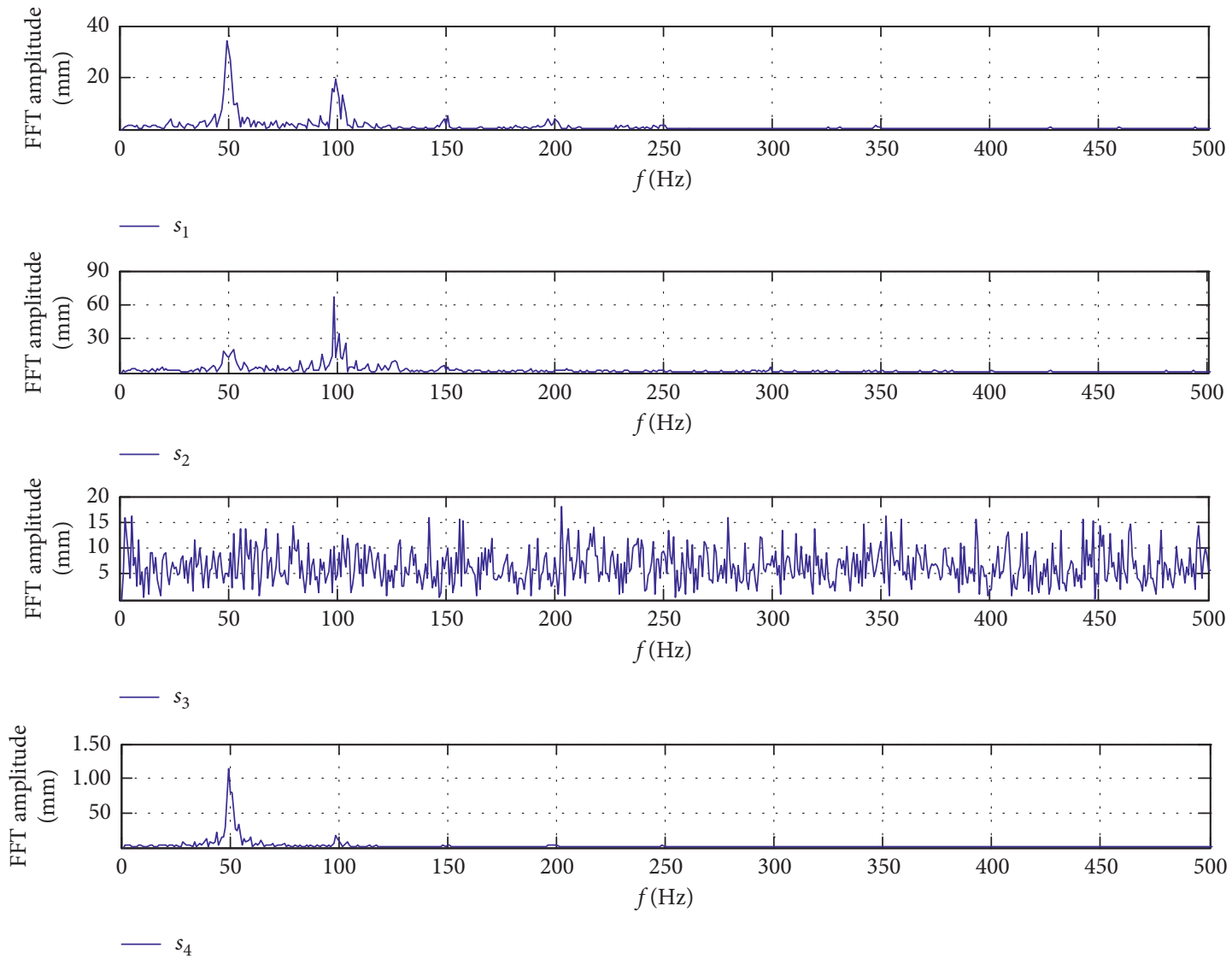

FIgURE 9: FFT of the separated signal using GAW-PSO.

feature. The energy of the separated signal S1 is mainly concentrated on $50 \mathrm{~Hz}$ and $100 \mathrm{~Hz}$, and the energy of other frequency components is very small, which is the characteristic of rotor unbalance fault. The frequency components of the separated signal S2 are concentrated on $50 \mathrm{~Hz}$ and $100 \mathrm{~Hz}$, but the second frequency amplitude is larger than the fundamental frequency, which corresponds to the rubimpact fault characteristics of the rotor. The energy comparison method of S3 is dispersive, which corresponds to the characteristics of random noise. The energy of the separated signal S4 is mainly concentrated at $50 \mathrm{~Hz}$, which corresponds to the power frequency of the rotor system.

\section{Conclusions}

In the actual blind source separation of rotor system fault signals, the blind source separation problem of random and dynamic mixing is more practical. Aiming at this problem, this paper proposed a blind separation method based on GAW-PSO for fault signals of the dynamic rotor system. This method adaptively adjusts the inertia weight by the change of fitness value before and after each particle iteration, eliminates the adverse effect of inertia component on the next iteration, and effectively enhances the performance of the algorithm. At the same time, genetic algorithm hybridization mechanism is introduced to increase the diversity of the population, which makes the particles not easy to fall into local optimum, and effectively solves the problem of blind separation of bearing signals with stochastic and dynamic mixing. On this basis, the algorithm complexity can be effectively reduced by parameterizing the separated orthogonal matrix. The experimental results showed that the GAW-PSO algorithm can effectively separate the dynamic mixed analog rotor fault signals, and the separation performance is better than PSO algorithm. The method is applied to the blind separation of the actual rotor system fault dynamic signals, which further verifies the feasibility of the method and effectively detects the coupling fault of the rotor system.

\section{Data Availability}

The data used to support the findings of this study are included within the article.

\section{Conflicts of Interest}

The authors declare that there are no conflicts of interest.

\section{Acknowledgments}

The authors are grateful to the Foundation of Henan Educational Committee (16A470021) and Key Scientific and Technological Project of Henan Province (172102210097) for the financial support of the current work. 


\section{References}

[1] Y. Yang, Z. Y. He, Z. Z. Li et al., "ASTFA-BSS method and its application in composite fault diagnosis for gearbox," China Mechanical Engineering, vol. 26, no. 15, pp. 2051-2056, 2015.

[2] F. Miao and R. Z. Zhao, "Separating for nonlinear mixed rotor fault signals with violent pulse interferences," Journal of Vibration, Measuremente Diagnosis, vol. 34, no. 4, pp. 625-630, 2014.

[3] H. Wang, S. Li, L. Song, L. Cui, and P. Wang, "An enhanced intelligent diagnosis method based on multi-sensor image fusion via improved deep learning network," IEEE Transactions on Instrumentation and Measurement, vol. 99, p. 1, 2019.

[4] Z. Meng, Z. Ma, D. Liu et al., "Blind source separation based on wavelet semi-soft threshold denoising," China Mechanical Engineering, vol. 27, no. 3, pp. 337-342, 2016.

[5] S. Q. Chen, Y. H. Shen, and K. Xu, "RLS for blind source separation using complexity pursuit," Journal of System Simulation, vol. 21, no. 3, pp. 658-660, 2009.

[6] Z. Li, X. Yan, Z. Tian, C. Yuan, Z. Peng, and L. Li, "Blind vibration component separation and nonlinear feature extraction applied to the nonstationary vibration signals for the gearbox multi-fault diagnosis," Measurement, vol. 46, no. 1, pp. 259-271, 2013.

[7] X. Xu, X. L. Liu, Z. L. Jiang et al., "Multi-sensor distributed fault detection method based on subjective bayesian reasoning," Journal of Mechanical Engineering, vol. 51, no. 7, pp. 91-98, 2015.

[8] Z. Meng and Z. Liang, "Fault diagnosis method for single channel rotating machinery based on EMMD and BSS," Chinese Journal of Scientific Instrument, vol. 34, no. 3, pp. 635-642, 2013.

[9] W. J. Ren and L. Tao, "Research on pump-jack fault diagnosis method based on particle swarm optimization," Journal of System Simulation, vol. 24, no. 2, pp. 482-487, 2012.

[10] M. Pal, R. Roy, J. Basu et al., "Blind source separation: a review and analysis," in Proceedings of the Oriental Cocosda Held Jointly with 2013 Conference on Asian Spoken Language Research and Evaluation, pp. 1-5, Gurgaon, India, 2013.

[11] C. Ji, K. Yang, Y. M. Tao et al., "An adaptive variable step-size blind source separation algorithm in non-stationary environment," Control and Decision, vol. 31, no. 4, pp. 735-639, 2016.

[12] Y. H. Shi and R. C. Eberhaert, "A modified particle swarm optimizer," in Proceedings of the IEEE Congress on Evolutionary Computation (CEC 1998), IEEE, Piscataway, NJ, USA, pp. 69-73, 1998.

[13] W. Deng, R. Yao, H. Zhao, X. Yang, and G. Li, “A novel intelligent diagnosis method using optimal LS-SVM with improved PSO algorithm," Soft Computing, vol. 23, no. 7, pp. 2445-2462, 2019.

[14] B. Wang, X. Zhang, C. Sun, and X. Chen, "A quantitative intelligent diagnosis method for early weak faults of aviation high-speed bearings," ISA Transactions, vol. 93, pp. 370-383, 2019.

[15] M. Sorensen, F. Van Eeghem, and L. De Lathauwer, "Blind multichannel deconvolution and convolutive extensions of canonical polyadic and block term decompositions," IEEE Transactions on Signal Processing, vol. 65, no. 15, pp. 41324145, 2017.

[16] B. Z. Ma, T. Q. Zhang, X. L. Jiang et al., "Blind source separation of multi-frequency-hopping signals based on adaptive inertia weight PSO algorithm," Telecommunication Engineering, vol. 56, no. 6, pp. 675-680, 2016.
[17] Y. Tang and J. P. Li, "A new algorithm of ICA: using the parametrized orthogonal matrixes of any dimensions," Acta Automatica Sinia, vol. 34, no. 1, pp. 31-39, 2008.

[18] G. C. Amaral, A. Baldivieso, J. D. Garcia et al., "A low-frequency tone sweep method for in-service fault location in subcarrier multiplexed optical fiber networks," Journal of Lightwave Technology, vol. 35, no. 10, pp. 2017-2025, 2017.

[19] M. S. Pedersen, J. Larsen, U. Kjems, and L. C. Parra, "A survey of convolutive blind source separation methods," in Handbook of Speech, pp. 1-34, Springer, New York, NY, USA, 2007.

[20] A. Hyvärinen and E. Oja, "A fast fixed-point algorithm for independent component analysis," Neural Computation, vol. 9, no. 7, pp. 1483-1492, 1997.

[21] A. Hyvarinen, "Fast and robust fixed-point algorithms for independent component analysis," IEEE Transactions on Neural Networks, vol. 10, no. 3, pp. 626-634, 1999.

[22] A. Cichocki and S. Amari, Adaptive Blind Signal and Image Processing, Wiley, New York, NY, USA, 2002. 